\title{
Yersinia pseudotuberculosis enterocolitis mimicking enteropathic $\gamma \delta$ T-cell lymphoma with abnormal clonality
}

Osamu Imataki ${ }^{1,3^{*}}$, Makiko Uemura ${ }^{1}$, Kensuke Matsumoto ${ }^{1}$ and Naoko Ishibashi ${ }^{2}$

\begin{abstract}
Background: Yersinia pseudotuberculosis generally infects the gastrointestinal tract and causes enteropathy symptoms suggesting infection. Y. pseudotuberculosis infections are often complicated with intraceliac lymphoadenopathy mimicking malignant lymphoma. This is a first case of Yersinia pseudotuberculosis enteropathy mimicking enteropathic $\gamma \delta$ T-cell lymphoma. This case highlighted the $\gamma \delta$ T-cell reaction to Yersinia enterocolitis sometimes mimicking malignant lymphoma clinically.
\end{abstract}

Case presentation: A 72-year-old female was referred to our institute due to abdominal pain with skin rush, fever and diarrhea. Computed tomography $(\mathrm{CT})$ scanning revealed mucosal swelling of the cecum with enlargement of regional lymph nodes. Laboratory data showed elevated CRP $(7.74 \mathrm{mg} / \mathrm{dL})$, an increased level of soluble interleukin-2 receptor (sIL-2R $3095 \mathrm{IU} / \mathrm{mL}$ ), and CD3+ $\gamma \delta$ T-cell circulation in peripheral blood and bone marrow (10.9\% and 3.9\%, respectively). Increased proportions of $\gamma \delta$ T-cells supported the diagnosis of malignant lymphoma. Colonoscopy demonstrated hemorrhagic mucosal erosion with partial ulceration, and the subsequent pathological findings at the inflammation site suggested malignant lymphoma histopathology in the colon. These objective findings were entirely consistent with enteropathic $\gamma \delta$ T-cell lymphoma. Thereafter, however, the microbiological results of the patient's stool at admission showed Yersinia pseudotuberculosis, and she was diagnosed as having Yersinia enterocolitis. All abnormal findings including subjective symptoms were in remission or mitigated within 2 weeks after her onset. Even the $\gamma \delta$ T-cell circulation disappeared ( $0.04 \%$ in peripheral blood), and we speculate that those cells were a reaction to the Yersinia infection.

Conclusion: In this case, a differential diagnosis included infectious enterocolitis from other immunogenic or malignant diseases. Although a measurement of sIL-2R is critical in differentiating malignant lymphoma in patients suffering with lymph adenopathy, that is not confirmative. This patient's case indicates that $T$ cells expressing the $\gamma \delta$ T-cell receptor might be associated with the acute and late phase reactions, in which T cells play a role in the construction of granulomas and the establishment of sequelae.

Keywords: Yersinia pseudotuberculosis, Yersinia enterocolitis, Enteropathic $ү \delta$ T-cell lymphoma malignant lymphoma

\footnotetext{
*Correspondence: oima@med.kagawa-u.ac.jp

'Division of Endocrinology and Metabolism, Hematology, Rheumatology and Respiratory Medicine, Department of Internal Medicine, Faculty of Medicine, Kagawa University, Kagawa, Japan

${ }^{3}$ Division of Hematology, Department of Internal Medicine, Faculty of

Medicine, Kagawa University, 1750-1 Ikenobe, Miki-cho, Kita-gun, Kagawa

761-0793, Japan

Full list of author information is available at the end of the article
}

\section{Biomed Central}

(c) 2014 Imataki et al.; licensee BioMed Central Ltd. This is an open access article distributed under the terms of the Creative Commons Attribution License (http://creativecommons.org/licenses/by/2.0), which permits unrestricted use, distribution, and reproduction in any medium, provided the original work is properly cited. 


\section{Background}

Yersinia pseudotuberculosis is a Gram-negative bacillus organism that can cause gastrointenstinal tract infections. Y. pseudotuberculosis infections are often complicated with intraceliac lymphoadenopathy in humans. Some cases progress to severe local inflammation with lymph node swelling, mimicking malignant lymphoma. In light of these characteristics, it is occasionally difficult to differentiate Yersinia enterocolitis from malignant lymphoma.

Most Yersinia infections arise from food contamination derived from water or raw pork. Notably, Y. pseudotuberculosis is known to cause the development of pseudotuberculotic mucosal lesions in the intestine or colon. The pathological features are characterized by an infiltration of various inflammatory cells, including lymphocytes and histiocytes, into necrotic granulomatous mucosa with reactive lymphoid hyperplasia.

\section{Case presentation}

A 72-year-old female was referred to our hospital after having experienced fever and abdominal pain for 5 days and a skin rash for 3 days before admission. She also complained of polyarthralgia, predominantly on her distal extremities. At admission, her body temperature was $38.8^{\circ} \mathrm{C}$ and she had a skin rash composed of exudative maculopapules and erythema multiforme over most of her body. Based on a consultation with a dermatologist who made a tentative diagnosis, we suspended loxoprofen and famotidine, medications she had been taking, because of the suspicion that the eruptions may have been drug-induced. Concomitantly, she received antibiotic therapy (meropenem) under adequate hydration, and underwent repeated blood and stool cultures. Laboratory data showed systemic inflammatory response represented by an increased $\mathrm{WBC}, 7380 / \mu \mathrm{L}$, and moderately elevated CRP, $7.74 \mathrm{mg} / \mathrm{dL}$. Her computed tomography (CT) scan demonstrated thickening of mucosa and enlarged mesenteric lymph nodes (Figure 1). A colonoscopy examination showed a round mucosal elevation with hemorrhagic erosion and ulceration at the

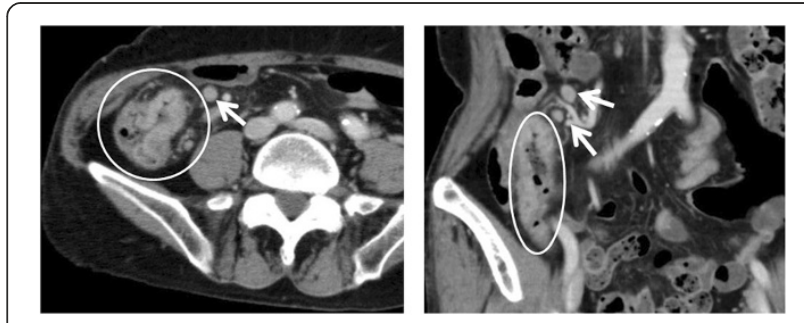

Figure 1 Her abdominal and pelvic enhanced CT revealed mucosal thickness of cecum (circle) and enlarged lesional lymph node (arrows). Left panel indicates transverse imaging and right panel indicates coronal imaging. terminal ileum and cecum (Figure 2), for which the pathological finding was suspicious malignant lymphoma with an infiltration of atypical lymphocytes into necrotic mucosa (Figure 3). Immunohistochemistry determined $\mathrm{CD}^{+}{ }^{+} \mathrm{T}$-cell distribution in the submucosal tissue, but did not confirm clonality of lymphocytes. Based on the pathology results, we performed an 18-fluoro-deoxyglucose positron emission tomography (FDG-PET)/CT scan, which drew an accumulation of FDG isotopes into regional mesenteric lymph nodes (SUV max 2.6). In addition, an increased level of soluble interleukin-2 receptor (sIL-2R), $3095 \mathrm{IU} / \mathrm{mL}$, and an expansion of a lymphocyte subset expressing $\gamma \delta$ T-cell receptor in peripheral blood and bone marrow $(10.9 \%$ and $3.9 \%$, respectively) supported the diagnosis of malignant lymphoma. These results led to a tentative diagnosis of enteropathy-associated T-cell lymphoma (EATL, or $\gamma \delta \mathrm{T}$-cell lymphoma). The patient's febrile and abdominal symptoms ameliorated rapidly a few days after antibiotics were initiated, and her skin eruptions improved over the following 7 days.

Since we could not obtain a confirmation of the pathological diagnosis, we reevaluated all aberrant clinical findings after the remission of the patient's symptoms. Within the 2-week treatment course, her laboratory data recovered to normal ranges. The sIL-2R level decreased to $483 \mathrm{IU} / \mathrm{mL}$, and $\gamma \delta \mathrm{T}$ cells in peripheral blood were almost nonexistent $(0.04 \%)$. A CT scan and colonoscopy conducted a few weeks after onset revealed slight swelling of the mesenteric lymph nodes and completely normal mucosa. During the second evaluation of the tentative diagnosis, Yersinia pseudotuberculosis was identified from her stool culture at admission. In light of all of the findings, we made the final diagnosis of Yersinia enterocolitis.

\section{Conclusion}

Most Yersinia infection is self-limiting pathogenesis, and in our patient the symptoms improved dramatically immediately after initiation of treatment with a broad antimicrobial, meropenem. Management of Yersinia enteroclolits requires antimicrobials and adequate symptom-based

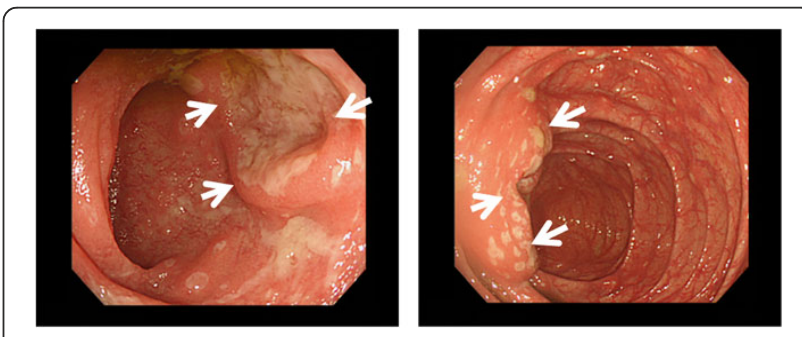

Figure 2 Colonoscopy showed hemorrhagic erosion and ulceration (arrows) at terminal ileum (left) and cecum (right). 

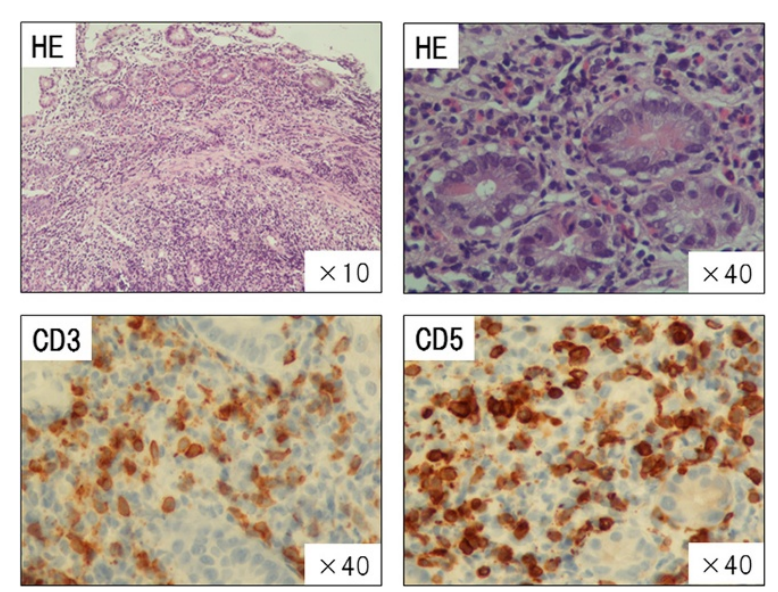

Figure 3 Histopathological findings were compatible of malignant lymphoma of colon mucosa, where small atypical lymphocytes (positive for CD3 and CD5) with cleaved nuclear infiltrate in intra-tubular submucosal area.

supportive care to avoid unfavorable complications such as dehydration. First-line drugs used against the bacterium include aminoglycosides and trimethoprim-sulfamethoxazole (TMP-SMZ). Microbiologically, pathogenesis of Yersinia depends on virulence against mucosal tissue that produces a necrotic consequence for the infected host. When encountering a patient with symptoms derived from febrile enterocolitis with or without skin eruption, clinicians should differentiate infectious enterocolitis from other immunogenic or malignant diseases. Causative pathogens of infectious enterocolitis presenting local inflammatory change at the cecum and terminal ileum include Mycobacterium tuberculosis, Salmonella (typhi and parachyphi), Campylobacter, and Yersinia. Invasive suppurative enterocolitis such as Yersinia can sometimes mimic other noninfectious pathogenicity, such as autoimmune diseases [1], inflammatory bowel diseases (Crohn's disease) [2] and malignancies [3]. In this case, a differential diagnosis included infectious enterocolitis from other immunogenic or malignant diseases. A measurement of sIL-2R is critical in differentiating malignant lymphoma in patients suffering with lymph adenopathy.

Over the past 20 years, a wide range of clinical manifestations of Yersinia enterocolitis has been revealed, and increased knowledge has been gained about this infection. The mechanism underlying the immunological reaction against Yersinia, however, remains obscure. The virulence of Yersinia organisms is associated with the enzymatic protein Yersinia protein kinase (Ypk), the production of which results in invasive suppurative infection of the lymph nodes [4]. The question of whether specific T lymphocytes are involved in the immune defense against Yersinia might be related to the mechanisms underlying this infection's extraintestinal manifestations and autoimmune-like sequelae, such as erythema nodosum, uveitis, and arthritis.

Autenrieth et al. demonstrated a specific T-cell reaction to Yersinia infection in a mouse-infection model [5]. In their phenotypic characterization of reactive T-cell clones on days 14, 20, and 24 after infection of Yersinia in C57BL/6 mice, they did not detect T cells positive for $\gamma \delta$ $\mathrm{T}$-cell receptors, but all $\mathrm{T}$ cells were positive for IL-2R. This result suggests that $\gamma \delta \mathrm{T}$ cells may no longer have been detectable in a subacute phase reaction at least 14 days after Yersinia infection. Autenrieth et al. isolated and identified specific T-cell clones at 12 to 14 days after restimulation by Yersinia antigens, and found that these $\mathrm{T}$ cells specifically produced a considerable amount of INF- $\gamma$ but not IL-2. These cells were both CD8 (Lyt2) or CD4 (L3T4)-positive. In fact, CD8 or CD4 T-cell induction by Yersinia infection is supported by the same mouse model [6]. In the Bühler study, the authors purified Yersinia enterocolitica microparticles, including invasive protein (invasin), and observed both enhanced CD4 and CD8 T-cell responses. They contended that invasion might promote a suitable inflammatory host response, and described the attractive possibility of an invasin vaccination against challenges by other pathogenic entero-organisms.

Although the deficiency of $\gamma \delta$ T-cell reaction for Yersinia infection was reported in the subacute infection mouse model, Young et al. demonstrated an expression of $\gamma \delta$ T-cell receptors 7 to 10 days after stimulation by Yersiniainfected autologous B cells [7]. They further elicited the killing ability of generated $\gamma \delta$ T-cell lines against Yersiniainfected target cells, even though $\gamma \delta$ T-cells were generated in concert with autologous synovial fluid mononuclear cells in a major histocompatibility complex (MHC)-independent manner. Young proposed that the participation and contribution of the $\gamma \delta$ T-cell response attenuates immunopathological mechanisms of reactive arthritis after Yersinia infection. From the Young experiment, we learned that in the early phase, 7-10 days after Yersinia infection, $\gamma \delta$ T-cells can proliferate and enhance local immunologic reaction in synovia.

In our patient, $\gamma \delta \mathrm{T}$ cells not only circulated in the peripheral blood but also infiltrated into bone marrow. There may be an effect of $\gamma \delta$ T cells on the establishment of tuberculosis-like or lymphoma-like pathology with inflammatory cell responses. Although the immunological role of this T-cell subset phenotypically positive for $\gamma \delta$ T-cell receptor is still under investigation, the reaction by these identical T-cell lines to the Yersinia organism would explain the multifocal pathology both in situ and at extraintestinal sites. This patient's case indicates that T cells expressing the $\gamma \delta \mathrm{T}$-cell receptor might be associated with the acute and late phase reactions, in which $\mathrm{T}$ cells play a role in the construction of granulomas and the establishment of sequelae. 


\section{Consent}

Written informed consent was obtained from the patient for publication of this case report and any accompanying images. A copy of the written consent is available for review by the Editor of this journal.

\section{Competing interests}

The authors declare that they have no competing interests.

\section{Authors' contributions}

Ol wrote the manuscript and made substantial contributions to the concept and design; $\mathrm{MU}$ was involved in drafting and supervising the manuscript; KM suggested important intellectual content and took part in the critical discussion; NI managed the study and reviewed the manuscript; all authors read and approved the final version of the manuscript.

\section{Author details}

'Division of Endocrinology and Metabolism, Hematology, Rheumatology and Respiratory Medicine, Department of Internal Medicine, Faculty of Medicine, Kagawa University, Kagawa, Japan. ${ }^{2}$ Postgraduate Medical Training Center, Faculty of Medicine, Kagawa University, Kagawa, Japan. ${ }^{3}$ Division of Hematology, Department of Internal Medicine, Faculty of Medicine, Kagawa University, 1750-1 Ikenobe, Miki-cho, Kita-gun, Kagawa 761-0793, Japan.

Received: 3 August 2013 Accepted: 18 January 2014

Published: 27 January 2014

\section{References}

1. Hassan SM, Doolittle BR: A case of Yersinia enterocolitica mimicking Kawasaki disease. Rheumatology (Oxford) 2009, 48(7):857-858.

2. Tuohy AM, O'Gorman M, Byington C, Reid B, Jackson WD: Yersinia enterocolitis mimicking Crohn's disease in a toddler. Pediatrics 1999, 104(3):e36.

3. Luedde T, Tacke F, Chavan A, Länger F, Klempnauer J, Manns MP: Yersinia infection mimicking recurrence of gastrointestinal stromal tumor. Scand J Gastroenterol 2004, 39(6):609-612.

4. Tafazoli F, Holmström A, Forsberg A, Magnusson KE: Apically exposed, tight junction-associated beta1-integrins allow binding and YopEmediated perturbation of epithelial barriers by wild-type Yersinia bacteria. Infect Immun 2000, 68(9):5335-5343.

5. Autenrieth IB, Tingle A, Reske-Kunz A, Heesemann J: T lymphocytes mediate protection against Yersinia enterocolitica in mice: characterization of murine T-cell clones specific for Y. enterocolitica. Infect Immun 1992, 60(3):1140-1149.

6. Bühler OT, Wiedig CA, Schmid Y, GrassI GA, Bohn E, Autenrieth IB: The Yersinia enterocolitica invasin protein promotes major histocompatibility complex class I- and class II-restricted T-cell responses. Infect Immun 2006, 74(7):4322-4329.

7. Young JL, Goodall JC, Beacock-Sharp H, Gaston JS: Human gamma delta T-cell recognition of Yersinia enterocolitica. Immunology 1997, 91(4):503-510.

Cite this article as: Imataki et al: Yersinia pseudotuberculosis enterocolitis mimicking enteropathic $\gamma \delta$ T-cell lymphoma with abnormal clonality. BMC Infectious Diseases 2014 14:42.

\section{Submit your next manuscript to BioMed Central and take full advantage of:}

- Convenient online submission

- Thorough peer review

- No space constraints or color figure charges

- Immediate publication on acceptance

- Inclusion in PubMed, CAS, Scopus and Google Scholar

- Research which is freely available for redistribution 